\title{
Perturbative nuclear physics
}

\author{
Silas R. Beane,,$^{1, *}$ David B. Kaplan, ${ }^{2,3, \dagger}$ and Aleksi Vuorinen ${ }^{4,5, \dagger}$ \\ ${ }^{1}$ Department of Physics, University of New Hampshire, Durham, New Hampshire 03824-3568, USA \\ ${ }^{2}$ Institute for Nuclear Theory, University of Washington, Seattle, Washington 98195-1550, USA \\ ${ }^{3}$ Instituto de Fisica Teorica, IFT-UAM/CSIC, Cantoblanco 28049, Spain \\ ${ }^{4}$ CERN, Physics Department, TH Unit, CH-1211 Geneva 23, Switzerland \\ ${ }^{5}$ Institut fur Theoretische Physik, TU Wien, Wiedner Hauptstr. 8-10, A-1040 Vienna, Austria
}

(Received 30 January 2009; published 14 July 2009)

\begin{abstract}
We present a new formulation of effective field theory for nucleon-nucleon $(N N)$ interactions that treats pion interactions perturbatively, and we offer evidence that the expansion converges satisfactorily to third order in the expansion, which we have computed analytically for $s$ - and $d$-wave $N N$ scattering. Starting with the Kaplan-Savage-Wise (KSW) expansion about the nontrivial fixed point corresponding to infinite $N N$ scattering length, we cure the convergence problems with that theory by modifying the short distance pion interaction, essentially resumming those contributions in the contact interactions. This method appears to make possible a host of high precision analytic few-body calculations in nuclear physics.
\end{abstract}

DOI: 10.1103/PhysRevC.80.011001

PACS number(s): 21.30.Fe, 12.39.Fe, 21.45.Bc

I. Introduction. All strong interactions in nuclear physics are of finite range and therefore should be amenable to an effective field theory (EFT) treatment at sufficiently low energy [1-3]. However, in contrast to the Fermi EFT for the weak interactions, the strong interactions between nucleons are nonperturbative even for momenta much smaller than the inverse range of the interactions; therefore the effect of the leading four-fermion interaction must be treated to all orders in perturbation theory, even though by conventional power counting it is an "irrelevant" operator. Weinberg [4-6] was the first to describe an EFT for nuclear forces and devised the prescription that one compute the nuclear potential in an EFT expansion, truncate at a given order, and then solve the Lippmann-Schwinger equation exactly with that potential. This program has since been pursued by a number of groups [7-23], with very impressive fits to phase shift data at $\mathrm{N}^{3} \mathrm{LO}$. An advantage of this approach is that the long distance part of the interaction correctly incorporates chiral symmetry; furthermore, with Weinberg's power counting scheme for the EFT expansion, there is in principle a systematic improvement of the results with increasing order. A disadvantage of Weinberg's scheme is that it is not renormalizable, in the sense that at any given order in the expansion there are divergences that cannot be absorbed by operators included at that order, arising from the singular nature of the EFT potential [24-26]. Thus results depend on a regulator scale $\Lambda$ that cannot be removed, implying that the treatment of short distance interactions is model dependent; in more recent developments the potential is regulated separately from the Lippmann-Schwinger equation, so that the result depends on two independent regularization scales [17]. An analysis of high partial wave channels at NLO in the Weinberg EFT in Ref. [26] demonstrated that the cutoff dependence was a

\footnotetext{
*silas@ physics.unh.edu

†dbkaplan@phys.washington.edu

${ }^{\ddagger}$ aleksi.vuorinen@cern.ch
}

feature of all channels subject to an attractive pion tensor force-despite the fact that there is no local operator to absorb this model dependence until order $(\ell+1)$ in the expansion for a channel with angular momentum $\ell$. Furthermore the analysis in Ref. [26] demonstrated that, at this order, observables in some channels (e.g. $\left.{ }^{3} P_{0}\right)$ are particularly sensitive to the value of the cutoff even at energies as low as $T_{\text {lab }}=50 \mathrm{MeV}$. It is argued that predictions at a given order only vary at the level of higher order corrections because the regulator is varied over some range, so that the model dependence does not interfere with the predictive power of the EFT. This hope is difficult to verify because the computations are all numerical, and the numerical evidence suggests that the acceptable range for $\Lambda$ is very narrow.

The alternative KSW theory entails an expansion of the $N N$ scattering amplitude, instead of the nuclear potential, effected by computing a well-defined class of Feynman diagrams at each order in the expansion [24,30,31]. KSW power counting is not determined by how operators scale near the trivial IR fixed point of the nucleon contact interaction (as in Fermi's weak interaction EFT); instead it is determined by operator scaling about the nontrivial UV fixed point corresponding to infinite scattering length. At this fixed point nucleon operators for $s$-wave scattering develop large anomalous dimensions and are resummed nonperturbatively, a reasonable starting point given how much larger $N N$ scattering lengths are than the range of their interaction. (See Ref. [32] for a related expansion utilizing explicit dibaryon fields [33].)

The KSW scheme expands the $N N$ scattering amplitude in powers of $Q$, where the nucleon momentum $p$, the pion mass $m_{\pi}$, and the inverse scattering length $1 / a$ are all considered $O(Q)$, while other mass scales such as the nucleon mass $M$ and the pion decay constant $f_{\pi}$ are taken to be $O(1)$. It was argued that convergence of the KSW expansion is governed by the scale $\Lambda_{N N}=16 \pi f_{\pi}^{2} /\left(g_{A}^{2} M\right)=300 \mathrm{MeV}$. An advantage of this approach is that the scattering amplitudes can be computed analytically, and at each order the amplitude is renormalized and independent of the cutoff. $N N$ phase shifts 


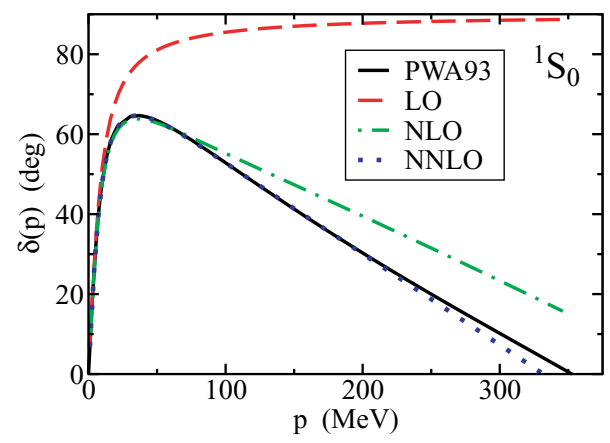

FIG. 1. (Color online) The ${ }^{1} S_{0} N N$ phase shift in the KSW expansion versus momentum in the center of mass frame to NNLO, compared with the Nijmegen PWA93 partial wave analysis [27]. Our calculation reproduces the result of Refs. [28] and [29].

were computed to order NNLO in Refs. [28] and [29]; the result for the spin-singlet ${ }^{1} S_{0}$ phase shift is shown in Fig. 1, plotted versus the momentum $p$ of each nucleon in the center of mass frame.

Although successful in the spin-singlet channel, it was discovered in Ref. [29] that the KSW expansion does not converge in the ${ }^{3} S_{1}$ channel, and the authors identified the singular tensor potential mediated by pions, scaling as $-1 / r^{3}$ for small $r$, to be the cause of the failure. Such a singular attractive interaction is incapable of supporting a ground state and no contact interaction can remedy this pathology. One possible solution suggested in Ref. [25] is to expand around the chiral $\left(m_{\pi}=0\right)$ limit, treating the infinite number of bound states in the pion potential as being short range and outside the purview of the EFT. In this article we propose a different solution: we modify the pion propagator in a manner reminiscent of Pauli-Villars regulation characterized by a heavy mass scale $\lambda$. This modification tames the $1 / r^{3}$ singularity in pion exchange, effectively shifting that physics into the contact interactions and reordering the summation of strong short-distance effects. The advantages of the KSW expansion are retained: there is a well-defined power counting scheme that organizes the calculation, and results are analytic. Dependence on the scale $\lambda$ can therefore be studied analytically, and we find that all contributions that grow as powers of $\lambda$ are absorbed into counterterms. The limit $\lambda \rightarrow \infty$ is therefore smooth, and the KSW expansion is recovered in that limit. Here we present promising results for the low-lying spin-triplet phase shifts to NNLO that indicate convergence of the expansion, and we discuss how the scale $\lambda$ resembles the renormalization scale $\mu$ encountered in perturbative QCD calculations: an unphysical scale that controls the ordering of the perturbative expansion and its convergence.

II. Short-distance modification of the pion propagator. Our starting point is the assumption that the failure of the $\mathrm{KSW}$ expansion is due to the singular short-distance pion tensor interaction, which can be eliminated by a shift in the contact interactions of the EFT. The underlying principles of EFT imply that we are free to distort the short-range pion interactions however we please, because the counterterms serve to ensure the correct low energy effects of short-distance physics. We therefore choose the modification to (i) make it possible to analytically perform the diagrammatic expansion and (ii) leave unaltered the KSW expansion of the spinsinglet channel, because apparently no convergence problem is encountered there. These considerations led us to replace the pion propagator $G_{\pi}\left(\mathbf{q}, m_{\pi}\right)$ by

$$
G_{\pi}\left(\mathbf{q}, m_{\pi}\right)+G_{(1,1)}(\mathbf{q}, \lambda)+G_{(1,0)}(\mathbf{q}, \lambda),
$$

where the subscript $(I, J)$ indicates the isospin and spin of a fictitious meson. Including couplings at the ends of the propagators, these expressions are given by

$$
\begin{aligned}
G_{\pi}\left(\mathbf{q}, m_{\pi}\right) & =i \frac{g_{A}^{2}}{4 f_{\pi}^{2}} \frac{\left(\mathbf{q} \cdot \boldsymbol{\sigma}_{1}\right)\left(\mathbf{q} \cdot \boldsymbol{\sigma}_{2}\right)\left(\boldsymbol{\tau}_{1} \cdot \boldsymbol{\tau}_{2}\right)}{\mathbf{q}^{2}+\mathbf{m}_{\pi}^{2}} \\
G_{(1,0)}(\mathbf{q}, \lambda) & =i \frac{g_{A}^{2} \lambda^{2}}{4 f_{\pi}^{2}} \frac{\left(\boldsymbol{\tau}_{1} \cdot \boldsymbol{\tau}_{2}\right)}{\mathbf{q}^{2}+\lambda^{2}},
\end{aligned}
$$

and

$$
G_{(1,1)}(\mathbf{q}, \lambda)=-G_{\pi}(\mathbf{q}, \lambda) .
$$

The $G_{(1,1)}$ term looks like exchange of a pion with the wrong sign propagator and mass $\lambda$, canceling the short distance $1 / r^{3}$ part of the pion-induced tensor interaction for $r \lesssim 2 \pi / \lambda$. The $G_{(1,0)}$ term is included to exactly cancel $G_{(1,1)}$ (up to a contact interaction) in the spin-singlet channel; it resembles the exchange of an $I=1, J=0$ meson, also of mass $\lambda$. In the above expressions $g_{A} \simeq 1.25$ and $f_{\pi} \simeq 93 \mathrm{MeV} ; \boldsymbol{\sigma}$ and $\boldsymbol{\tau}$ are spin and isospin matrices, respectively. Note that the only free parameter is the mass scale $\lambda$. We expect that for $\lambda \gtrsim 2 \Lambda_{N N}$ the derivative expansion is not adversely affected and that the original KSW expansion is recovered in the $\lambda \rightarrow \infty$ limit.

We emphasize that we are not using $G_{(1,1)}$ and $G_{(1,0)}$ to model real meson exchange, but only as a device to eliminate the strong short-distance behavior from the tensor pion exchange, putting all that physics in the contact interactions that are fit to data. Choosing the masses in $G_{(1,1)}$ and $G_{(1,0)}$ to both equal $\lambda$ greatly simplifies the analytic computations.

III. NNLO calculation of spin-triplet amplitudes. Making use of the modified pion propagator Eq. (1) and classifying the mass scale $\lambda$ to also be $O(Q)$, we have computed all the Feynman diagrams in Refs. [28] and [29] relevant for the ${ }^{3} S_{1},{ }^{3} D_{1}$, and $\epsilon_{1}$ partial wave channels. These diagrams are evaluated using dimensional regularization and we choose the renormalization scale, $\mu=m_{\pi}$. The analytic formulas for our NNLO calculations will be given elsewhere; here we present the results graphically. In Fig. 2 we show our results with $\lambda=750 \mathrm{MeV}$ for the ${ }^{3} S_{1},{ }^{3} D_{1}$ and $\epsilon_{1}$ phase shifts, compared with the Nijmegen partial wave analysis [27]. All three of our results are improvements over the NNLO KSW computation in Refs. [28] and [29] and, with the exception of $\epsilon_{1}$, show signs of converging on the correct answer. The result for $\epsilon_{1}$ is less convincing, but it should be noted that the anomalously small value for $\epsilon_{1}$ in nature suggests that delicate cancellations are at play, and one would only expect an EFT prediction to start converging at high order in the expansion.

The dependence of our results on $\lambda$ is displayed in Fig. 3, where the bands indicate the changes in the phase shifts over the range $600 \mathrm{MeV} \leqslant \lambda \leqslant 1000 \mathrm{MeV}$. It is apparent from these figures that our results are not extremely sensitive at low $p$ to the value we take for $\lambda$. 

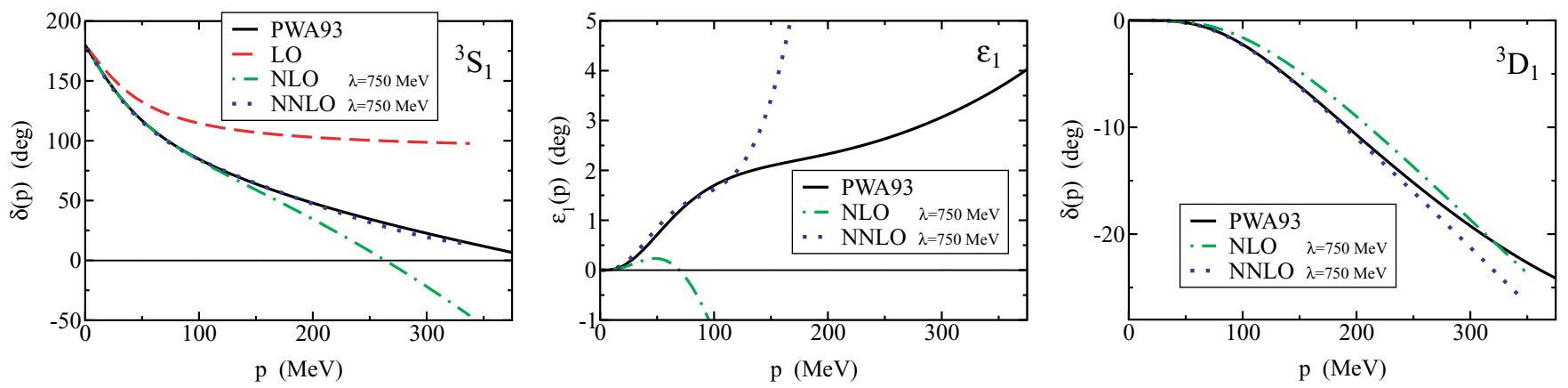

FIG. 2. (Color online) New results for the ${ }^{3} S_{1},{ }^{3} D_{1}$, and $\bar{\epsilon}_{1}$ phase shifts plotted versus momentum in the center of mass frame to NNLO, compared with the Nijmegen PWA93 partial wave analysis.

The role of the scale $\lambda$ in these calculations can be easily addressed given the analytic form we have derived for the scattering amplitudes. It may seem strange that $\lambda-\mathrm{a}$ regularization scale-is being treated as $O(Q)$, which is our low energy expansion scale. In particular, one might worry that scattering amplitudes have terms proportional to powers of $\lambda / \Lambda_{N N}$, which is formally $O(Q)$ but numerically $>1$. In fact though one can show analytically that, at each order in the expansion, contributions to the amplitudes proportional to positive powers of $\lambda$ are all absorbed into the counterterms available at that order. Therefore the amplitudes only depend on inverse powers of $\lambda$, and in the $\lambda \rightarrow \infty$ limit the fictitious meson propagators in Eq. (1) decouple and one smoothly recovers the results of Refs. [28] and [29].

IV. Discussion. The EFT scheme we have presented here for computing $N N$ scattering in perturbation theory appears to converge well and preserve the desirable feature of the KSW scheme that at each order the amplitude can be computed as a well-defined set of Feynman diagrams. Unlike the KSW scheme, there is now a new dimensionful parameter $\lambda$ that regulates the short-distance tensor interaction. The manner with which we have performed this regulation is certainly not unique, and we have shown that our results are not particularly sensitive to the value of $\lambda$ and that over a wide range for $\lambda$ the variation of the phase shifts are comparable to or smaller than higher order corrections in the EFT expansion.

However, we know that by taking $\lambda \rightarrow \infty$ we recover the KSW expansion, which fails to converge above $p \sim 100 \mathrm{MeV}$.
The parameter $\lambda$ apparently plays a role analogous to the renormalization scale $\mu$ in perturbative QCD. The scale $\mu$ is unphysical, and a nonperturbative QCD calculation will not depend on it; however, at any finite order in perturbation theory, amplitudes do depend on $\mu$, and varying $\mu$ corresponds to reordering the perturbative expansion. Choosing $\mu$ appropriately (e.g., via the BLM scale-setting prescription [34]) can optimize the perturbative expansion, while nonoptimal choices for $\mu$ lead to poor convergence. Similarly, $\lambda$ is an unphysical parameter, and varying $\lambda$ constitutes a reordering of the the EFT expansion, with smaller $\lambda$ resulting in more of the pion interaction being accounted for in the resummed contact interactions. Taking $\lambda \simeq 750 \mathrm{MeV}$ appears to optimize the expansion, while choosing $\lambda=\infty$ yields the standard $\mathrm{KSW}$ expansion, which fails to converge at relatively low momenta.

It is not possible to directly compare our expansion with the Weinberg expansion results at a given order, because the calculations are arranged differently. For example, one-pion and two-pion exchange appear at NLO and $\mathrm{N}^{3} \mathrm{LO}$ in the KSW expansion, respectively, while they appear at LO and NLO in the Weinberg expansion. Nevertheless, numerically our NNLO results compare favorably with the NLO Weinberg expansion results in Ref. [14], with the exception of $\epsilon_{1}$, which is comparable to LO.

In a subsequent paper we will present the detailed form of the amplitudes at NNLO for the partial waves presented here, as well as extending our work to other channels. While we
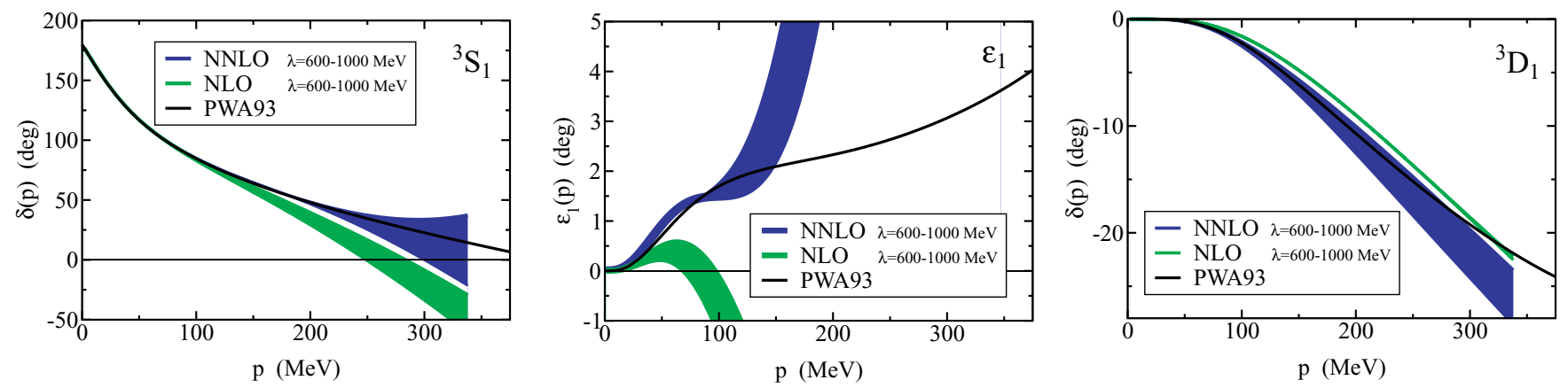

FIG. 3. (Color online) The NLO (green band) and NNLO (blue band) results for the ${ }^{3} S_{1},{ }^{3} D_{1}$, and $\bar{\epsilon}_{1}$ phase shifts showing their variation as $\lambda$ is varied in the range $600 \mathrm{MeV} \leqslant \lambda \leqslant 1000 \mathrm{MeV}$. 
have demonstrated that our expansion fixes the convergence problems at NNLO in the $s$ and $d$ waves that had been found in Ref. [29], we have not addressed here the issues raised in Ref. [26] concerning the need for counterterms in higher partial wave channels with an attractive tensor force. While our expansion is fully renormalized and therefore does not lack the necessary counterterms at any order to make the amplitude cutoff independent, it is possible that these channels (such as ${ }^{3} P_{0}$ ) may not converge well until the order at which the first counterterm appears. To study this will require computing the $p$-wave amplitudes to N3LO, a project which is currently under way.

Our theory provides a well-defined prescription for computing a number of additional processes to NNLO, such as electromagnetic effects, including form factors, Compton scattering, polarizabilities, and radiative capture, and it will be interesting to compare such results with experiment as additional tests of the efficacy of this method.

We thank U. van Kolck and M. J. Savage for useful communications. DBK thanks the Instituto de Fisica Téorica of the Universidad Autónoma de Madrid for hospitality during part of this project. SRB is partly supported by NSF CAREER Grant PHY-0645570. DBK was supported in part by the DOE under Contract DE-FGO3-00ER41132, by Spanish MEC Grant SAB2006-0089 and Project FPA2006-05423, and by the regional Comunidad de Madrid HEPHACOS Project. AV was supported in part by the Austrian Science Foundation, FWF, Project M1006, as well as the Sofja Kovalevskaja Grant of the Humboldt Foundation.
[1] S. R. Beane, P. F. Bedaque, W. C. Haxton, D. R. Phillips, and M. J. Savage, At the Frontier of Particle Physics: Handbook of $Q C D$, Vol. 4, edited by M. Shifman (World Scientific Publishing Company, 2002).

[2] P. F. Bedaque and U. van Kolck, Annu. Rev. Nucl. Part. Sci. 52, 339 (2002).

[3] D. B. Kaplan, Five Lectures on Effective Field Theory (2005), arXiv:nucl-th/0510023.

[4] S. Weinberg, Phys. Lett. B251, 288 (1990).

[5] S. Weinberg, Nucl. Phys. B363, 3 (1991).

[6] S. Weinberg, Phys. Lett. B295, 114 (1992).

[7] C. Ordoñez and U. van Kolck, Phys. Lett. B291, 459 (1992).

[8] C. Ordoñez, L. Ray, and U. van Kolck, Phys. Rev. Lett. 72, 1982 (1994).

[9] U. van Kolck, Phys. Rev. C 49, 2932 (1994).

[10] C. Ordoñez, L. Ray, and U. van Kolck, Phys. Rev. C 53, 2086 (1996).

[11] J. L. Friar, D. Huber, and U. van Kolck, Phys. Rev. C 59, 53 (1999).

[12] M. C. M. Rentmeester, R. G. E. Timmermans, J. L. Friar, and J. J. de Swart, Phys. Rev. Lett. 82, 4992 (1999).

[13] V. Bernard, N. Kaiser, and U.-G. Meissner, Nucl. Phys. A615, 483 (1997).

[14] E. Epelbaum, W. Gloeckle, and U.-G. Meissner, Nucl. Phys. A671, 295 (2000).

[15] E. Epelbaum et al., Phys. Rev. Lett. 86, 4787 (2001).

[16] E. Epelbaum et al., Eur. Phys. J. A 15, 543 (2002).

[17] E. Epelbaum, W. Gloeckle, and U.-G. Meissner, Eur. Phys. J. A 19, 401 (2004).
[18] E. Epelbaum, W. Glockle, and U.-G. Meissner, Nucl. Phys. A747, 362 (2005).

[19] D. R. Entem and R. Machleidt, Phys. Lett. B524, 93 (2002).

[20] D. R. Entem and R. Machleidt, Phys. Rev. C 66, 014002 (2002).

[21] D. R. Entem and R. Machleidt, Phys. Rev. C 68, 041001(R) (2003).

[22] M. Pavon Valderrama and E. Ruiz Arriola, Phys. Rev. C 74, 054001 (2006).

[23] M. Pavon Valderrama and E. Ruiz Arriola, Phys. Rev. C 74, 064004 (2006).

[24] D. B. Kaplan, M. J. Savage, and M. B. Wise, Nucl. Phys. B478, 629 (1996).

[25] S. R. Beane, P. F. Bedaque, M. J. Savage, and U. van Kolck, Nucl. Phys. A700, 377 (2002).

[26] A. Nogga, R. G. E. Timmermans, and U. van Kolck, Phys. Rev. C 72, 054006 (2005).

[27] V. G. J. Stoks, R. A. M. Klomp, M. C. M. Rentmeester, and J. J. de Swart, Phys. Rev. C 48, 792 (1993).

[28] S. Fleming, T. Mehen, and I. W. Stewart, Phys. Rev. C 61, 044005 (2000).

[29] S. Fleming, T. Mehen, and I. W. Stewart, Nucl. Phys. A677, 313 (2000).

[30] D. B. Kaplan, M. J. Savage, and M. B. Wise, Nucl. Phys. B534, 329 (1998).

[31] D. B. Kaplan, M. J. Savage, and M. B. Wise, Phys. Lett. B424, 390 (1998).

[32] J. Soto and J. Tarrus, Phys. Rev. C 78, 024003 (2008).

[33] D. B. Kaplan, Nucl. Phys. B494, 471 (1997).

[34] S. J. Brodsky, G. P. Lepage, and P. B. Mackenzie, Phys. Rev. D 28, 228 (1983). 